\title{
Chaotic swimming of phoretic self-propelled particles
}

\author{
Wei-Fan Hu, ${ }^{1}$ Te-Sheng Lin, ${ }^{2}$ Salima Rafai, ${ }^{3}$ and Chaouqi Misbah ${ }^{3, *}$ \\ ${ }^{1}$ Department of Applied Mathematics, National Chung Hsing University, 145 Xingda Road, Taichung 402, Taiwan \\ ${ }^{2}$ Department of Applied Mathematics, National Chiao Tung University, 1001 Ta Hsueh Road, Hsinchu 300, Taiwan \\ ${ }^{3}$ Univ. Grenoble Alpes, CNRS, LIPhy, F-38000 Grenoble, France
}

(Dated: July 24, 2019)

\begin{abstract}
Swimming of a rigid phoretic particle in an isotropic fluid is studied numerically as a function of the dimensionless solute emission rate (or Péclet number $P e$ ). The particle sets into motion at a critical $P e$. Whereas the particle trajectory is straight at small enough $P e$, it is found that it looses its stability at a critical $P e$ in favor of a meandering motion. When $P e$ is increased further the particle meanders at short scale but its trajectory wraps into a circle at larger scale. Increasing even further $P e$ causes the swimmer to escape momentarily the circular trajectory in favor of chaotic motion lasting for a certain time, before regaining a circular trajectory, and so on. The chaotic bursts become more and more frequent as $P e$ increases, until the trajectory becomes fully chaotic, via intermittency scenario. The statistics of the trajectory is found to be of run and tumble-like nature at short enough time, and of diffusive nature at long time without any source of noise.
\end{abstract}

Introduction.- The field of microswimmers covers today an extremely wide panel of systems going from prokaryotic and eukaryotic microorganisms (such as bacteria, algae, leukocytes, and so on) to artificial microswimmers [1,2]. A prototypical example of the later is Janus-like particle, named after the two-faced Roman god, their motion originates from the asymmetry of their surface properties. Other popular designs of artificial swimmers consists of active droplets [3-7], involving a chemical reaction, or a constant local source/sink of the solute. In these circumstances a spontaneous symmetry breaking of the solute distribution field on the surface (or in the bulk) may emerge in the form of a solute polarity giving rise to hydrodynamical flow (of Marangoni type) leading to self-propulsion.

Several studies have been devoted to the elucidation of the mechanisms of such Marangoni driven propulsion $[8,9]$. In particular, the onset of self propulsion has been described in terms of a coupling between a chemical reaction that produces a surface tension gradient at the surface of the droplet and a mechanism of advection that allows to sustain this gradient against diffusion. The description of the surface activity of the droplet involves in general advection-diffusion-reaction contributions for each species involved in the system (for a recent review, see [10]). Combined theoretical and numerical efforts have allowed elucidating the basic elements at the origin of the occurence of spontaneous locomotion $[3,4,6,9]$. The outcome is that beyond a critical value of a dimensionless solute emission compared to diffusion (the Péclet number $P e$ ) the droplet sets into a spontaneous unidirectional motion. However, not only straight propulsion but also meandering trajectories have been observed in different experiments [11-14].

So far, the explanation of complex motions, such as circular and helical trajectories, relies on the complexity of the swimming particle (e.g. chiral shape) [12] or that of the suspending medium, such as viscoelasticty [14]. These various rich behaviors raise naturally the question of whether or not this complexity may already be hidden in purely isotropic media due to the intrinsic nonlinearities of the problem. This is the main objective of this Letter. We show that circular tra- jectories appear in the full model without any restriction on the model symmetry. Moreover, a transition to chaos via intermittency scenario is observed and quantified. The chaotic behavior can lead to a run and tumble-like dynamics of purely deterministic origin.

The model.- We consider a two-dimensional circular shaped particle of radius $a$ immersed in a fluid of dynamic viscosity $\eta$. The fluid is assumed to be incompressible and obeys the Stokes equations. Surrounding solute particles are emitted or adsorbed on the particle with isotropic emission rate $\mathscr{A}$. The solute interacts with the particle through a short-range potential with a characteristic length $\lambda$ that is much smaller than $a$. In the so-called sharp interface limit this amounts to a slip tangential velocity [6] on the particle surface (see below). The concentration of the solute, denoted by $c$, diffuse with diffusivity $D$ and is advected by the fluid flow.

As in [6], the length, fluid velocity, the concentration, and the pressure are scaled by the characteristic values $a$, $|\mathscr{A} \mathscr{M}| / D, a|\mathscr{A}| / D$, and $\eta|\mathscr{A} \mathscr{M}| / a D$ respectively. Here $\mathscr{M}= \pm k_{B} T \lambda^{2} / \eta$ is the signed mobility (see [15]) defined with the Boltzmann constant $k_{B}$ and the temperature $T$. The model equations in dimensionless form are described in the following: In a co-moving frame attached to the particle center the tangential slip velocity on the particle surface is given, in a polar coordinate system, by $\mathbf{u}(1, \theta)=M \nabla_{s} c$, where $\mathbf{u}(r, \theta)$ is the fluid velocity, $\nabla_{s}=(\partial / \partial \theta) \tau$ (where $\tau$ is the unit tangent vector) is the surface gradient operator and $M=\mathscr{M} /|\mathscr{M}|= \pm 1$ is the dimensionless mobility. In the far field $(r \rightarrow \infty)$, the velocity field converges to the translational phoretic velocity $-\mathbf{U}$. The solute concentration $c(r, \theta, t)$ is governed by the following advection-diffusion equation and the Stokes equation

$$
\frac{\partial c}{\partial t}+\mathbf{u} \cdot \nabla c=\frac{1}{P e} \Delta c, \Delta \mathbf{u}-\nabla p=\mathbf{0}, \nabla \cdot \mathbf{u}=0
$$

where the Péclet number is defined by $P e=|\mathscr{A} \mathscr{M}| a / D^{2}$. The associated boundary condition of surface activity reads $\frac{\partial c}{\partial r}(1, \theta, t)=-A$, where $A=\mathscr{A} /|\mathscr{A}|= \pm 1$ is the dimensionless emission rate $(A>0$ : emission, $A<0$ : adsorption). One 
should note that, in most previous studies that considered the three-dimensional cases, the fluid domain is unbounded and the concentration is assumed to attenuate in the far-field limit $(c \rightarrow 0$ as $r \rightarrow \infty$ ). However, such an assumption is not appropriate in $2 \mathrm{D}$. Indeed, it is known that, in $2 \mathrm{D}$ the solute transport equation (1) for $P e=0$ with the far-field attenuation condition does not support steady-state solutions due to the logarithm divergence [16]. In order to cure this problem, we assume that the size of the fluid domain is a finite number $R$ and the attenuation condition is thus replaced by the outer Dirichlet boundary condition $c(R, \theta, t)=0$. A preliminary 3D study recovers the occurence of complex motion, presented below.

The force-free constraint on the solid particle (using the reciprocal theorem) to relate the phoretic velocity $\mathbf{U}(t)$ to the slip velocity (and thus to concentration from the above slip condition) [17]:

$$
\mathbf{U}(t)=-\frac{1}{2 \pi} \int_{0}^{2 \pi} M \nabla_{s} c(1, \theta, t) \mathrm{d} \theta .
$$

Since the concentration $c$ is periodic in $\theta$-direction, it can be expressed by Fourier series as

$$
c(r, \theta, t)=\sum_{k=-\infty}^{\infty} \hat{c}_{k}(r, t) e^{i k \theta},
$$

where $\hat{c}_{k}$ are the Fourier modes. Substituting this representation into Eq. (2), one finds (in Cartesian components):

$$
\mathbf{U}(t)=M\left[-\operatorname{Re}\left(\hat{c}_{1}(1, t)\right), \operatorname{Im}\left(\hat{c}_{1}(1, t)\right)\right],
$$

where Re and Im denote real and imaginary parts, respectively. The velocity field can be expressed as $\mathbf{u}=\left(\frac{1}{r} \frac{\partial \psi}{\partial \theta},-\frac{\partial \psi}{\partial r}\right)$ (in polar components), where $\psi$ is the stream function and has the following analytical form $[18,19]$

$$
\psi(r, \theta, t)=\sum_{k=-\infty}^{\infty} \frac{1-r^{2}}{2 r^{|k|}} i k M \hat{c}_{k}(1, t) e^{i k \theta} .
$$

where we have used the boundary condition $\mathbf{u}(1, \theta)=M \nabla_{s} c$ to express the series coefficients in terms of $c$. Thus, the velocity $\mathbf{u}$ (as well as are $\mathbf{U}$ ) is given in terms of $c$, and when injected into (1) yields a closed nonlinear equation for $c$. Solution (4) is valid for infinite system, but for a finite size $R=200$ the residual errors are of order 0.005 (see [20]). In the following we will see how the full model, not restricted to symmetric solutions, leads to the emergence of rich dynamics, turning into chaos at large Péclet numbers.

Stationary solution and linear stability analysis.- A stationary solution where there is no net flow and zero phoretic velocity $\mathbf{U}(t) \equiv 0$ exists at all Péclet numbers with the solute concentration $c_{0}(r)=A \ln (R / r)$. We perform a linear stability analysis of this solution by introducing an infinitesimal perturbation $\phi(r, \theta, t)=\sum_{k=-\infty}^{\infty} \hat{\phi}_{k}(r, t) e^{i k \theta}$ with $\phi \ll c_{0}$. Neglecting higher order terms, the following relation for the Fourier mode $\hat{\phi}_{k}$ is obtained:

$$
\frac{\partial \hat{\phi}_{k}}{\partial t}=-A M k^{2} \frac{1-r^{2}}{2 r^{|k|+2}} \hat{\phi}_{k}(1, t)+\frac{1}{P e}\left(\frac{\partial^{2}}{\partial r^{2}}+\frac{1}{r} \frac{\partial}{\partial r}-\frac{k^{2}}{r^{2}}\right) \hat{\phi}_{k} .
$$

Since $r>1$, the coefficient of the first term on the right hand side of the equation is positive/negative when $A$ and $M$ have the same/opposite sign, respectively. That is, the first term may be stabilizing or destabilizing, while the second term represents diffusion that is always stabilizing. Whether or not a small perturbation of the concentration is able to trigger the spontaneous symmetry-breaking swimming motion depends on the competition between these two mechanisms. In the case when $A$ and $M$ have opposite signs any fluctuation acting on the stationary solution should die out and hence for any Péclet number there is only one observable solution that is stationary. Henceforth, we will concentrate on the nontrivial case where $A$ and $M$ have the same sign. For definitness we set $A=M=1$.

We first perform the linear stability of the stationary solution. The eigenvalue problem reads $\sigma_{k} \hat{\phi}_{k}=\mathbf{L}_{k} \hat{\phi}_{k}$, where $\sigma_{k}$ is the eigenvalue (we look for perturbations in the form $e^{\sigma_{k} t}$ ) and $\mathbf{L}_{k}$ is the linear operator on the right hand side of Eq. (5). The eigenvalue problem is solved numerically using a Chebyshevspectral method. In all simulations, we set the size of the outer domain $R=200$. It is found that, for $k=1$, one of the eigenvalues becomes positive at $P e \approx 0.466$ so that the stationary solution loses its stability at this $P e$ value. This marks the transition between a nonmotile to motile particle. The mode $k=1$ corresponds to polarity of the concentration field.

Spontaneous symmetry-breaking autophoretic motion, $\mathrm{Pe}>$ $P e_{1}$. - Once the instability threshold is reached $\left(P e_{1} \sim\right.$ $2 / \ln (R)$; see the full expression in [20]) nonlinear terms are necessary in order to fix the amplitude of the swimming speed. This task is handled numerically. We employ the second-order Runge-Kutta time-marching scheme while spatial derivatives in $r$-direction are performed either by second-order finite difference method or Chebyshev spectral differentiation, derivatives in $\theta$-direction are achieved through Fourier spectral differentiation. In the present time-stepping method, the nonlinear advection term is treated in an explicit manner while the diffusion term is solved implicitly (see [21] for more detail about the fast solver of the present scheme).

A symmetry-breaking solution occurs when $P e \geq P e_{1} \simeq$ 0.466 . We find that in the range $0.466 \lesssim P e \lesssim 4.65$ the particle swims in a given direction (fixed by initial conditions) with constant velocity. The concentration distribution at $P e=3$, exhibiting a comet-like pattern. In this case, there exists a nonzero concentration gradient on the particle surface and the particle propels itself in a straight direction with the constant phoretic velocity $\|\mathbf{U}\| \approx 0.148$.

Figure 1 shows the phoretic velocity as a function of the Péclet number. The particle sets into a directed motion in the form of a supecritical bifurcation. Note that a non-monotonic variation of the phoretic velocity is observed in the range $0.466 \lesssim P e \lesssim 4.65$ and the particle attains the highest swimming speed at the optimal value of $P e \approx 2.1$. This result is in qualitative agreement with that found in [6] for threedimensional axisymmetric phoretic particles.

Meandering motion, $\mathrm{Pe}>\mathrm{Pe}_{2}$.- - The directed swimming solution looses its stability (a secondary instability) at $P e_{2} \approx$ 


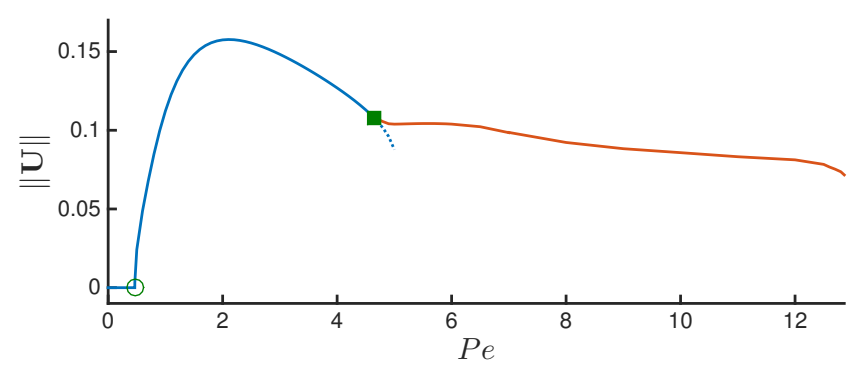

FIG. 1. The bifurcation diagram of the phoretic velocity $\|\mathbf{U}\|$ as a function of $P e$. The empty circle indicates the bifurcation point $P e_{1}=$ 0.466 and the filled-square indicates the bifurcation point $P e_{2}=4.65$. The dashed line corresponds to a steady-state but unstable straight motion and the red line to non straight motions (see below).
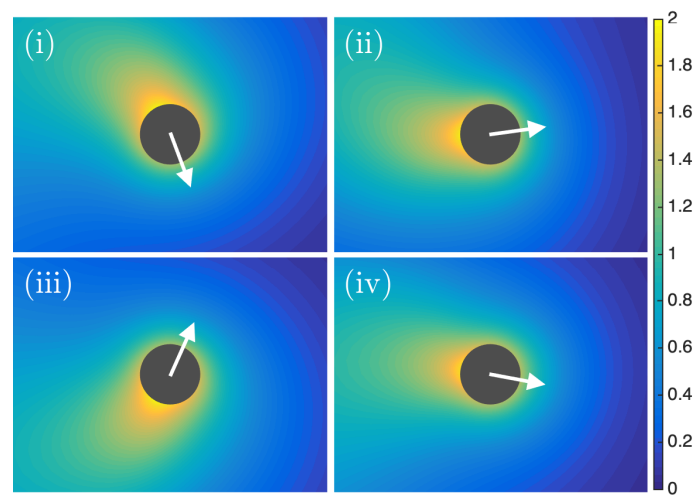

FIG. 2. Snapshots of the bead and distribution of the solute concentration for $P e=6$ undergoing meandering motion, white arrow showing the direction of the motion. The color coding shows the magnitude of the concentration and the arrow indicates the swimming direction.

4.65 in favor of a meandering motion: the particle follows a meandering path as shown in Fig. 3 (a). In this regime the swimming velocity is no longer a constant but periodically oscillates in time. As an example, we show in Fig. 2 the time-periodic concentration distribution at $P e=6$. These solutions are shown as the red solid line in Fig. 1. For such time-periodic solution the swimming speed is defined by $\|\mathbf{U}\|=T^{-1} \int_{0}^{T}\|\mathbf{U}(t)\| d t$, which is measured over one time period, $T$. This transverse instability is the first initial stage for a more complex dynamics, as discussed below. The meandering period scales as $a D /(A M)$, which is a compromise between diffusion, flow mobility along the surface and the emission rate. An interesting feature has emerged: the meandering swimmer exhibits a higher velocity than the directed one. Although the directed motion is unstable, we can still follow this branch by solving the steady-state problem (a steady directed motion always exist, though it becomes unstable) by imposing to the particle to set at the same rectilinear direction. By this manner, we could determine its velocity. The dashed line shown in Fig. 1 indicates the unstable equilibrium solution corresponding to a directional movement. The meandering solution swims faster than the directed one, presumably due to subtle nonlinear effects.For $P e \simeq P e_{2}$ the period of meandering diverges as $\left(\mathrm{Pe}-\mathrm{Pe}_{2}\right)^{1 / 2}$, which is symptomatic of a supercritical bifurcation (see [20]) .
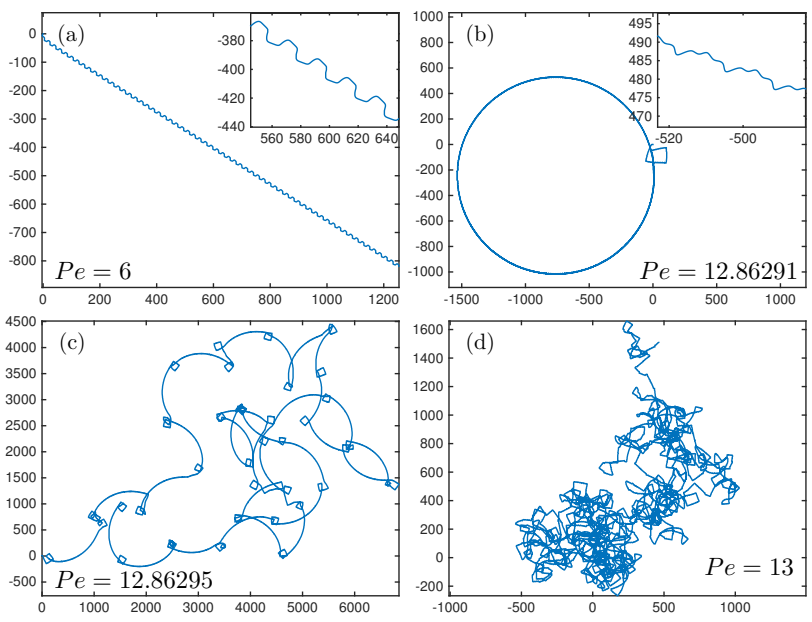

FIG. 3. Different trajectories: meander, circular motion with short scale meander, intermittency and chaos.

Circular motion, $\mathrm{Pe}>\mathrm{Pe}_{3}$. - By increasing the Péclet number above $P e_{3} \simeq 12.5145$ we find that the above meandering solution becomes unstable in a somewhat remarkable way (in fact, a period doubling bifurcation occurs at $P_{3}$ ). Figure 2 shows the bead with an asymmetric concentration field leading to trajectory deviation. As seen in Fig. 3(b) for the case of $P e=12.86291$, although the local movement is again zigzaglike (or meander) at short scale, the velocity direction continuously varies with time, and the global dynamics of swimming pattern converges to a circle for the long time behavior (radius is insensitive to system size [20]). The particle short scale meandering is maintained, whereas a longer scale dynamics drives the particle trajectory to wrap around a circular trajectory. Note that here the studied system is fully isotropic in a marked contrast with the observed complex trajectories (like meander, circles, and so on) for chiral particles [12]. Here, the present complex motion emerges from the inherent nonlinear advection-diffusion coupling. This coupling leads to an axial symmetry-breaking bifurcation of the comet (Fig. 1), so that in the bead frame the comet drift sideways along the bead surface leading to a circular trajectory. The radius of the circle diverges at $\mathrm{Pe}_{3}$ (and so does the period of revolution) like $\left(P e-P_{3}\right)^{-\alpha}$, with $\alpha \simeq 0.2$, acting as a macroscopic length scale (a coherence-like length) much larger than the swimmer size. Further development would be needed in order to explain the numerical exponent ( $\alpha \sim 0.2$; see Fig. S1 in [20]).

Chaotic swimming motion, $\mathrm{Pe}>\mathrm{Pe}_{4} \simeq 12.86293$. - Increasing the Péclet number even further reveals another instability: the particle quits the circular trajectory and enters into an apparently chaotic regime, where it exhibits few runs and tumbles during some time, before it regains a circular trajectory (Fig. 3(c)). This type of chaotic bursts appears in a non- 
regular manner, as also shown in Fig. 4 which represents the swimming speed of the particle close to the transition towards chaos. This nonregularity is due to the subcritical nature of the bifurcation towards chaos. Indeed, during a certain time the motion is regular, and the degree of natural intrinsic fluctuations can cause the system to jump from the regular solution to the apparently chaotic one. As the Péclet number is increased further and further the chaotic bursts become more and more frequent, until a critical value $P e_{4} \simeq 13$ where the whole trajectory becomes erratic in time. In Fig. 1 we shows the magnitude of the averaged velocity as a function of $\mathrm{Pe}$. This is symptomatic of the intermittency scenario [22]. Its particularity is that the limit cycle (in our case the meandering-circular regular solution) looses its stability at a given critical value of $\mathrm{Pe}$ in a subcritical fashion (the analogue of a first order transition for systems at equilibrium). That is, the regular solution may coexist with new solutions and the particle can jump back and forth from one solution to the other, when higher modes $(k>1)$ approaches the instability conditions. This manifests itself in temporary chaotic bursts until coexisting solutions (the analogue of metastability in equilibrium systems) is fully lost and the regime becomes fully chaotic.

In the chaotic regime, the particle exhibits an apparently random motion (of purely deterministic origin), see Fig. 3(d). To quantify this specific random walk, we measure the mean square displacement

$$
\operatorname{MSD}(\tau)=<\|\mathbf{r}(t+\tau)-\mathbf{r}(t)\|^{2}>,
$$

where $\mathbf{r}(t)$ is the location of the particle at time $t$ and $<\cdot\rangle$ denotes the average along the entire trajectory. Figure 5 reports the mean square displacement (msd) corresponding to the trajectory at $P e=13$. At short times the particle produces a persistent swimming motion and msd is then quadratic in time. At longer times, a de-correlation process due to chaotic turns takes place with msd proportional to $t$, typical of a classical random walk. Actually, it is not obvious that a chaotic motion is equivalent (at long time) to normal diffusion. There are several chaotic maps yielding anomalous diffusion [23]. This is attributed to the topology of the Poincaré map, in which the trajectory may spend long time periods in some regions of phase space. Despite that chaos via intermittency favors long period stay in specific regions of phase space [22], a diffusionlike behavior still persists here.

Discussion.- We have identified the emergence of complex dynamics going from straight swimming to chaotic trajectories for a rigid particle in a purely isotropic fluid medium. Chaos is also found in our preliminary 3D study[20]). This highlights the fact that the complexity can arise from a minimal version. The chaotic regime can be quantified as runand-tumble-like events. The first unstable mode is $k=1$, that provides directed swimming. As $P e$ increases higher harmonics becomes unstable, first $k=2$ and $k=3$, and so on. The activity of these three modes is sufficient to generate chaos. Interestingly, $P e_{k}(k>1)$ are independent of system size $R$.

The time over which the diffusion-like behavior is reached (as well as the duration of the circulation trajectory) are much

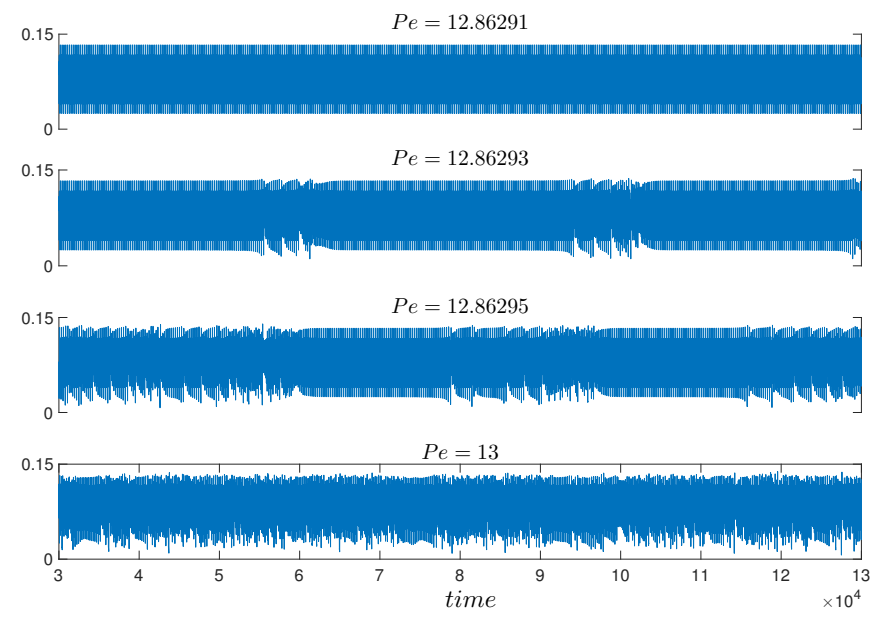

FIG. 4. The swimming speed $\|\mathbf{U}(t)\|$ from regular meander to chaos. Chaotic bursts are more and more frequent as $P e$ increases.

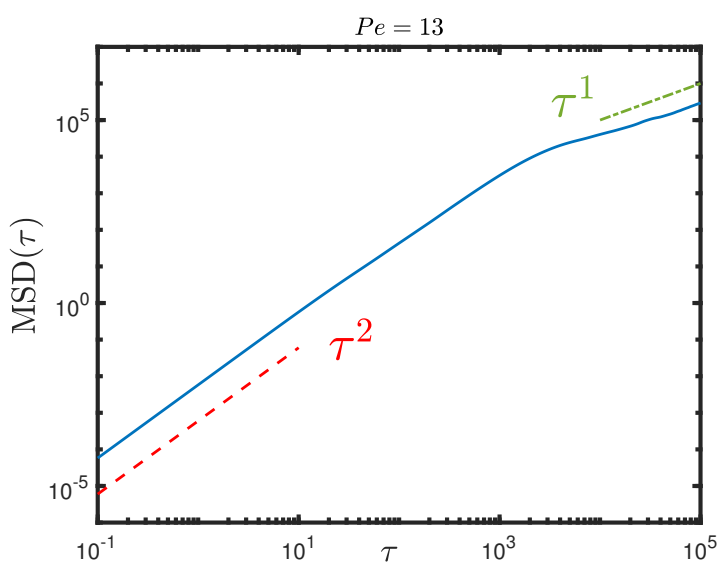

FIG. 5. Mean square displacement of a particle in the chaotic regime at $P e=13$.

larger than the typical time for the growth of the linear modes $\sim 1 / \sigma_{1}$. This feature may be attributed to the very nature of chaos via intermittency. Indeed, in this regime it is known that trajectory in phase space alternates intermittently between slow regular motion close to the marginally stable fixed point (the regular solution), and chaotic bursts. As a consequence, the correlation decay may exhibit long range behavior. From physical point of view, the longtime behavior may be related to the persistence of circular orbits (albeit transients) having a radius much larger than the particle radius

Non trivial motions have been observed so far with complex shapes or in complex media $[12,14]$. The main message of this Letter is the demonstration that a complex motion is possible in an isotopic, nonviscoelastic phoretic system. Experiments on non colloidal systems (autophoretic drops)[7] have provided some evidence of complex trajectories without diging further into their properties. The present results may serve as a guide for future systematic analyses. 
We thank CNES (Centre National d'Etudes Spatiales) (C.M.) and the French-German university program "Living Fluids" (grant CFDA-Q1-14) (C.M. and S.R.). W.-F. Hu acknowledges support by Ministry of Science and Technology, Taiwan under research grant MOST-107-2115-M-005004-MY2. T.-S. Lin acknowledges support from MOST-1072115-M-009-008-MY2.

* chaouqi.misbah@univ-grenoble-alpes.fr

[1] E. Lauga and T. Powers, Rep. Prog. Phys. 72, 096601 (2009).

[2] M. C. Marchetti, J.-F. Joanny, S. Ramaswamy, T. B. Liverpool, J. Prost, M. Rao, and R. A. Simha, Reviews of Modern Physics 85, 1143 (2013).

[3] A. Y. Rednikov, Y. S. Ryazantsev, and M. G. Velarde, Physics of Fluids 6, 451 (1994).

[4] A. Golovin, Y. P. Gupalo, and Y. S. Ryazantsev, Journal of Applied Mechanics and Technical Physics 30, 602 (1989).

[5] S. Thutupalli, R. Seemann, and S. Herminghaus, New Journal of Physics 13, 073021 (2011).

[6] S. Michelin, E. Lauga, and D. Bartolo, Phys. Fluids 25, 061701 (2013).

[7] Z. Izri, M. N. Van Der Linden, S. Michelin, and O. Dauchot, Phys. Rev. Lett. 113, 248302 (2014).

[8] M. Schmitt and H. Stark, EPL (Europhysics Letters) 101, 44008 (2013).
[9] M. Morozov and S. Michelin, The Journal of chemical physics 150, 044110 (2019).

[10] C. C. Maass, C. Krüger, S. Herminghaus, and C. Bahr, Annual Review of Condensed Matter Physics 7, 171 (2016).

[11] C. Krüger, G. Klös, C. Bahr, and C. C. Maass, Phys. Rev. Lett. 117, 048003 (2016).

[12] H. Löwen, The European Physical Journal Special Topics 225, 2319 (2016).

[13] M. Suga, S. Suda, M. Ichikawa, and Y. Kimura, Phys. Rev. E 97, 062703 (2018).

[14] N. Narinder, C. Bechinger, and J. R. Gomez-Solano, Phys. Rev. Lett. 121, 078003 (2018).

[15] S. Michelin and E. Lauga, Journal of Fluid Mechanics 747, 572 (2014).

[16] E. Yariv, J. Fluid Mech. 816, R3 (2017).

[17] G. J. Elfring, Phys. Fluids Phys. Fluids 27, 023101 (2015).

[18] D. Sondak, C. Hawley, S. Heng, R. Vinsonhaler, E. Lauga, and J.-L. Thiffeault, Phys. Rev. E 94, 062606 (2016).

[19] J. Blake, Bulletin of the Australian Mathematical Society 5, 255 (1971).

[20] Supplementary Information containing (i) the scaling of the radius of the circular trajectory as a function of $P e$ and (ii) the evolution of the spectrum of different harmonics as a function of $\mathrm{Pe}$.

[21] M.-C. Lai and W.-C. Wang, Numer. Meth. Part Differ. Equ. 18, 56 (2002).

[22] P. Bergé, Y. Pomeau, and C. Vidal, Order within chaos: towards a deterministic approach to turbulence, A WileyInterscience publication (Paris, 1986).

[23] T. Geisel and J. Nierwetberg, Phys. Rev. Lett. 48, 7 (1982). 